\title{
Discrete Visible Luminescence of Helium Atoms and Molecules Desorbing from Helium Clusters: The Role of Electronic, Vibrational, and Rotational Energy Transfer
}

\author{
K. von Haeften, ${ }^{1}{ }^{*}$ A. R. B. de Castro, ${ }^{3}$ M. Joppien, ${ }^{2}$ L. Moussavizadeh, ${ }^{2}$ R. von Pietrowski, ${ }^{1}$ and T. Möller ${ }^{1}$ \\ ${ }^{1}$ Hamburger Synchrotronstrahlungslabor HASYLAB at Deutsches Elektronen Synchroton DESY, Hamburg, \\ Notkestr. 85, 22603 Hamburg, Germany \\ ${ }^{2}$ II. Institute für Experimentalphysik, Universität Hamburg, Germany \\ ${ }^{3}$ Laboratorio National de Luz Sincrotron, Campinas 13081-90, Brazil
}

(Received 24 March 1997)

\begin{abstract}
Discrete visible and near-infrared luminescence of a beam of photoexcited helium clusters is reported. The emission lines are attributed to free helium atoms and molecules desorbing from clusters in electronically excited states. Depending on the excitation energy, various atomic and molecular singlet and triplet states are involved in the relaxation process. With increasing cluster size the intensity of molecular transitions becomes dominant. The temperature of ejected molecules could be estimated to $T_{\mathrm{vib}} \sim 2500 \mathrm{~K}$ and $T_{\text {rot }} \sim 450 \mathrm{~K}$ and is much higher than that of the cluster itself. [S0031-9007(97)03267-5]
\end{abstract}

PACS numbers: 36.40.Mr, 33.50.Dq, 36.40.Qv

Visible and infrared (ir) luminescence spectroscopy is one of the oldest and most established methods for the exploration of electronic level structure and excited states dynamics of atoms and molecules. The derivation of the well known Rydberg formula which was based on the observation of luminescence spectra of metal atoms is one of the most prominent examples of its usefulness. Condensed matter physics has also benefited substantially from luminescence spectroscopy, but detailed information on the excited state dynamics of pure materials is often difficult to obtain for two reasons: (i) In most cases only rather broad luminescence bands due to transitions between the lowest electronically excited state and the ground state are observed. (ii) Transitions between electronically excited states of pure solids or liquids similar to that in atoms and molecules are usually not observed because nonradiative relaxation processes following electronic excitation depopulate the highly excited states extremely efficiently.

Liquid helium is presumably the only exception to this rule. In contrast to all other pure condensed materials liquid helium emits a very rich discrete spectrum in the ir which is due to transitions between electronically excited states of helium atoms or molecules enclosed in bubbles $[1,2]$. Thus, in the context of cluster science helium is an ideal material to study the excited state dynamics and radiative and nonradiative processes as a function of the cluster size.

In this Letter we report on the observation of visible and ir luminescence of helium cluster beams. Similar to $\mathrm{He}_{2}$ [3] and the bulk liquid [2], helium clusters emit a large number of discrete lines in the visible and infrared range. The excited states dynamics is investigated as a function of the cluster size and in addition the excitation energy is varied. This permits detailed insight into the relaxation pathway. Further, this is the first luminescence study of helium with photoexcitation at all, since all work done in the past on the excited states of atomic, molecular, or liquid helium has been performed with unspecific excitation using charged particles, e.g., electrons, protons, $\alpha$ particles. In general, nonradiative processes dominating the relaxation pathway depend on the particle density. In helium clusters the particle density depends strongly on the cluster size [4] which makes them even more interesting to study. The role of the two parameters size and density can be studied independently by preparing clusters of the different isotopes ${ }^{4} \mathrm{He}$ and ${ }^{3} \mathrm{He}$. Here we concentrate on results for the most abundant isotope ${ }^{4} \mathrm{He}$. Finally, it should be noted that clusters of ${ }^{4} \mathrm{He}$ containing more than a few hundred atoms have very interesting structural and thermodynamic properties like superfluidity [4]. This may also affect relaxation processes in clusters, e.g., desorption of species because atoms can change their position in the cluster very efficiently.

The measurements were performed at the experimental station CLULU at HASYLAB [5]. In brief, helium clusters are prepared in a free expansion of cold helium gas $(4-30 \mathrm{~K})$ at a stagnation pressure of $2000 \mathrm{mbar}$ through a nozzle of $60 \mu \mathrm{m}$ in diameter. The beam consisting of atoms and clusters is photoexcited with monochromatized, tunable synchrotron radiation in the energy range between 20-25 eV. Luminescence spectra in the visible and ir are recorded with a 0.275 m CzernyTurner spectrometer equipped with a liquid nitrogen cooled CCD camera. Overview spectra are taken with a 150 lines per $\mathrm{mm}$ grating. In the case of helium clusters a precise determination of the cluster size is very difficult because they are very fragile. Here we give only rough estimates based on results for heavier rare gas clusters and well known scaling laws [6].

The emission of dense gaseous and liquid helium consists of very broad and intense continuous emissions in the vacuum ultraviolet (VUV) and much weaker discrete lines and bands in the visible and the ir range [7]. The VUV continuum is assigned to bound-free luminescence between 
the first excited singlet state and the repulsive ground state. A similar continuum is also emitted from helium clusters [8]. In addition, visible and ir luminescence is observed which is much less intense due to the competition with the VUV luminescence.

Figure 1 shows visible and ir spectra of a helium cluster beam in comparison with a spectrum recorded on an atomic beam. The excitation energy is tuned to the $1 s^{1} S_{0} \rightarrow$ $3 p^{1} P_{1}$ atomic transition. At this energy the absorption of clusters is rather strong too [9]. As expected, in the case of the atomic beam the $3 p^{1} P_{1} \rightarrow 2 s^{1} S_{0}$ line, which is the only dipole allowed transition in this range, can be seen. Apart from this line, the spectrum of the cluster beam shows two other lines which coincide with the atomic $3 s^{1} S_{0} \rightarrow 2 p^{1} P_{1}$ and $3 d^{1} D_{2} \rightarrow 2 p^{1} P_{1}$ transition. Unlike the emissions of liquid helium [1], fluorescence lines of helium clusters are within error limits of $0.1 \mathrm{~nm}$ identical to that of free atoms and not broadened. Prominent transitions are given in Table I. We therefore conclude that the strong lines are emitted from free atoms desorbing from the cluster.

With increasing cluster size the $3 s^{1} S_{0} \rightarrow 2 p^{1} P_{1}$ and the $3 d^{1} D_{2} \rightarrow 2 p^{1} P_{1}$ gain in intensity compared to the $3 p^{1} P_{1} \rightarrow 2 s^{1} S_{0}$ atomic transition. In all measurements the emission from the $3 d^{1} D_{2}$ state being energetically closer to the $3 p^{1} P_{1}$ state dominates over that from the

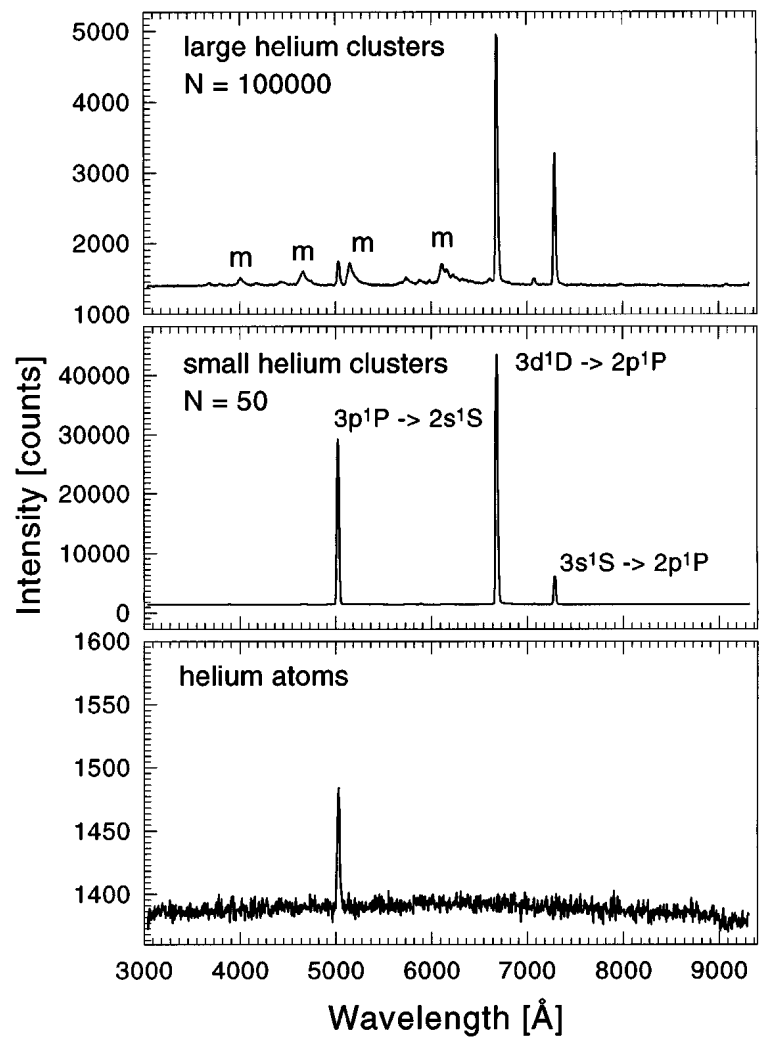

FIG. 1. Fluorescence spectra of helium clusters of different sizes. The excitation energy of $23.1 \mathrm{eV}$ is associated with the $1 s^{1} S_{0} \rightarrow 3 p^{1} P_{1}$ atomic transition. Molecular lines are indicated by $m$.
$3 s^{1} S_{0}$ state. Since the $3 s^{1} S_{0}$ state is $166 \mathrm{meV}$ and the $3 d^{1} D_{2}$ is $13 \mathrm{meV}$ [10] below the $3 p^{1} P_{1}$ state, this indicates that the nonradiative relaxation into lower lying states becomes more efficient with increasing cluster size.

Large clusters $(N=100000)$ also emit a large number of weak additional lines which can be attributed to emissions of free helium molecules. This trend is in agreement with the findings for bulk liquid helium for which molecular emissions are much stronger that the atomic transitions [1]. The origin of the molecular lines is visualized in Fig. 2. Discrete molecular emissions are due to transitions between strongly bound Rydberg states of $\mathrm{He}_{2}$. In the following we refer to centers if excited molecules or atoms are inside the cluster. Molecular centers are formed in the relaxation process in competition with atomic centers. However, there is an important difference between the emission from bulk liquid helium and clusters. In the first case atoms or molecules being enclosed in bubbles emit fluorescence light while in the latter case free atoms and molecules which are ejected from the cluster emit. The high efficiency of desorption can be explained in the following way. Atomic and molecular centers enclosed in a bubble have a high mobility in the cluster. Every time such a bubble approaches the cluster surface the excited atom or molecule is ejected as a result of a repulsive force between the excited electron and the surrounding atoms. A similar, well known process is responsible for the desorption of atoms and molecules from rare gas solids [11].

From potential energy curves (see Fig. 2) it is obvious that molecular centers in their lowest vibrational level are energetically favorable. The self-localization process of electronically excited centers usually starts with atomic or molecular centers in high vibrational levels. With increasing cluster size these centers need more time to reach the surface. Thus, atomic centers are converted into molecular centers more efficiently the larger the clusters are and the intensity of molecular emissions increases (Fig. 3).

Luminescence spectra of excited helium clusters containing on average 2500 atoms show a dependence on

TABLE I. Atomic and molecular transitions observed following excitation of helium clusters.

\begin{tabular}{cccc}
\hline \hline Atomic trans. & $\begin{array}{c}\text { Emission } \\
(\AA)\end{array}$ & Molecular trans. & $\begin{array}{c}\text { Band head } \\
(\AA)\end{array}$ \\
\hline $3 p^{1} P \rightarrow 2 s^{1} S$ & 5015.7 & $D^{1} \Sigma_{u}^{+} \rightarrow B^{1} \Pi_{g}$ & 6595.9 \\
$3 d^{1} D \rightarrow 2 p^{1} P$ & 6678.2 & $d^{3} \Sigma_{u}^{+} \rightarrow b^{3} \Pi_{g}$ & 6400.8 \\
$3 s^{1} S \rightarrow 2 p^{1} P$ & 7281.4 & $F^{1} \Sigma_{u}^{+} \rightarrow B^{1} \Pi_{g}$ & 6314.3 \\
$3 p^{3} P \rightarrow 2 s^{3} S$ & 3888.6 & $F^{1} \Pi_{u} \rightarrow B^{1} \Pi_{g}$ & 6246.8 \\
$3 d^{3} D \rightarrow 2 p^{3} P$ & 6678.2 & $F^{1} \Delta_{u} \rightarrow B^{1} \Pi_{g}$ & 6112.4 \\
$3 s^{3} S \rightarrow 2 p^{3} P$ & 7281.4 & $f^{3} \Sigma_{u}^{+} \rightarrow b^{3} \Pi_{g}$ & 5959.5 \\
$4 p^{1} P \rightarrow 2 s^{1} S$ & 3964.7 & $f^{3} \Pi_{u} \rightarrow b^{3} \Pi_{g}$ & 5887.5 \\
$4 d^{1} D \rightarrow 2 p^{1} P$ & 4921.9 & $f^{3} \Delta_{u} \rightarrow b^{3} \Pi_{g}$ & 5734.9 \\
$4 s^{1} S \rightarrow 2 p^{1} P$ & 5047.7 & $E^{1} \Pi_{g} \rightarrow A^{1} \Sigma_{u}^{+}$ & 5134.5 \\
$4 p^{3} P \rightarrow 2 s^{3} S$ & 3187.7 & $e^{3} \Pi_{g} \rightarrow a^{3} \Sigma_{u}^{+}$ & 4649.6 \\
$4 d^{3} D \rightarrow 2 p^{3} P$ & 4471.5 & $I^{1} \Pi_{g} \rightarrow A^{1} \Sigma_{u}^{+}$ & 4003.8 \\
$4 s^{3} S \rightarrow 2 p^{3} P$ & 4713.1 & $i^{3} \Pi_{g} \rightarrow a^{3} \Sigma_{u}^{+}$ & 3677.4 \\
\hline \hline
\end{tabular}




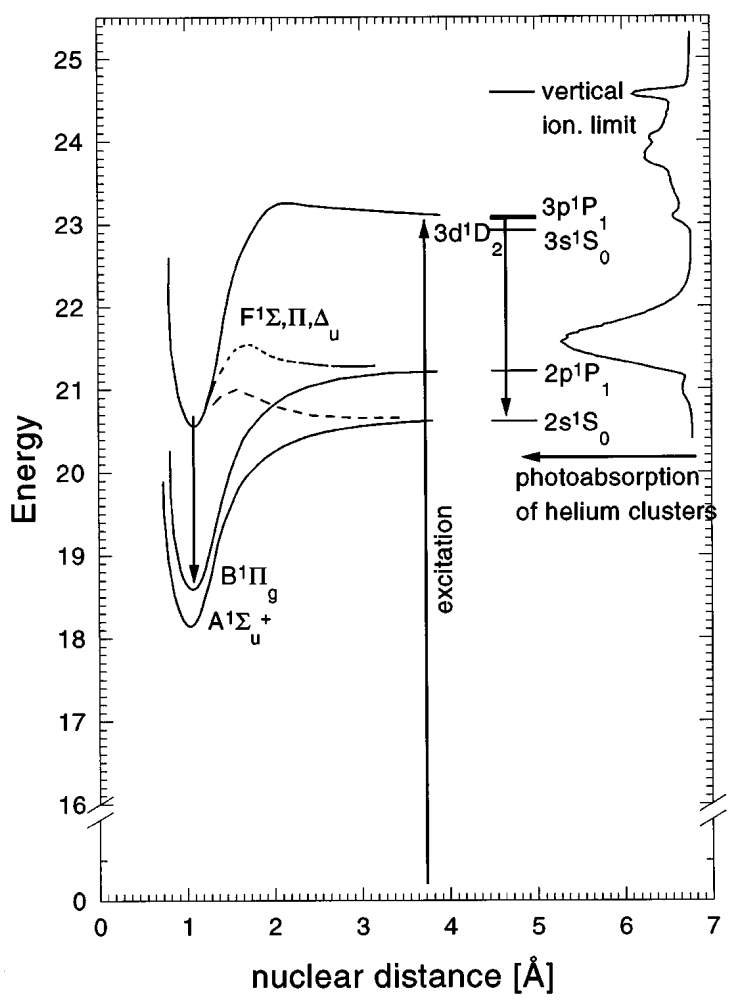

FIG. 2. Energy transfer within photoexcited helium clusters. The potential curves of $\mathrm{He}_{2}$ are plotted using data of Refs. [9] and [14].

excitation energy (see Fig. 4). The excitation energy has been tuned to absorption bands of the clusters, in other words, atoms which are always present in the background gas are not directly excited. Three general trends are observed:

(i) With increasing excitation energy the number of lines increases. These lines are due to transitions from highly excited states. This indicates that nonradiative processes which usually lead to an efficient depopulation

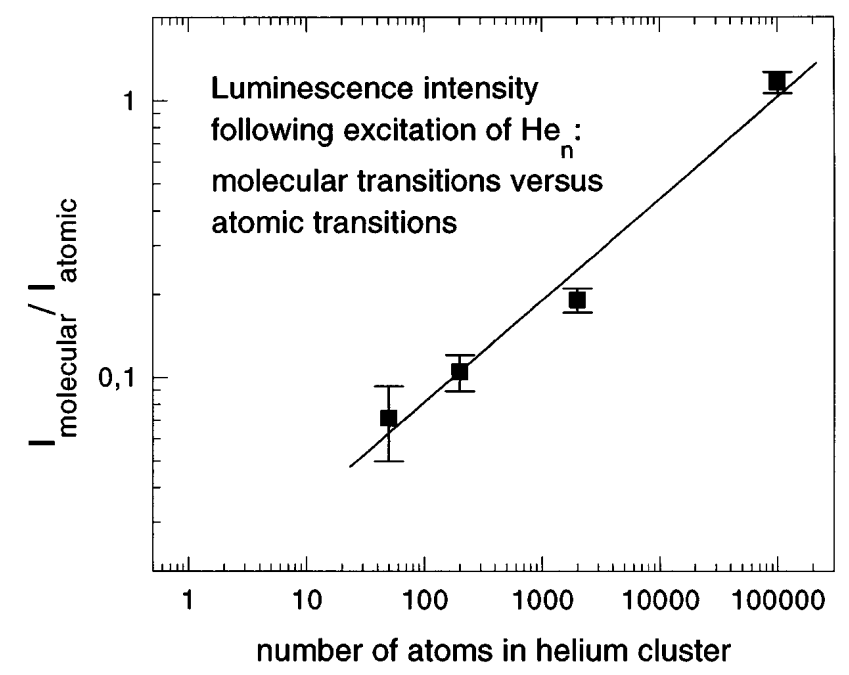

FIG. 3. Ratio between the intensities of all detected atomic and molecular lines. of highly excited states [7] are slow, or at least not much faster than radiative relaxation processes.

(ii) Molecular emissions gain in intensity relative to atomic emissions with increasing excitation energy. It is reasonable to assume that electronically excited atomic centers are formed in the first step of the relaxation cascade. These excited atomic centers can relax into bound molecular states. However, they have to tunnel through potential barriers. Thus, the intensity of molecular centers should be related to the vibrational relaxation rate in the long range part of the molecular potential close to the atomic dissociation limit. The height of the barrier (see Fig. 2) between the atomic dissociation limit and the tightly bound part of the potential curve decreases with the principal quantum number [12]. It is therefore reasonable that with increasing excitation energy tunneling through this barrier, vibrational relaxation and hence the formation of $\mathrm{He}_{2}$ becomes more likely as observed.

(iii) Interestingly, lines of the atomic and molecular triplet system can be seen. The intensity of these lines becomes stronger the higher the principal quantum numbers of the excited states are. The presence of triplet lines is not expected because spin-orbit interaction in helium is very weak and hence the conversion of electron spin should be forbidden. Indeed, the photoexcitation spectrum of helium atoms and clusters does not show any triplet line [9].

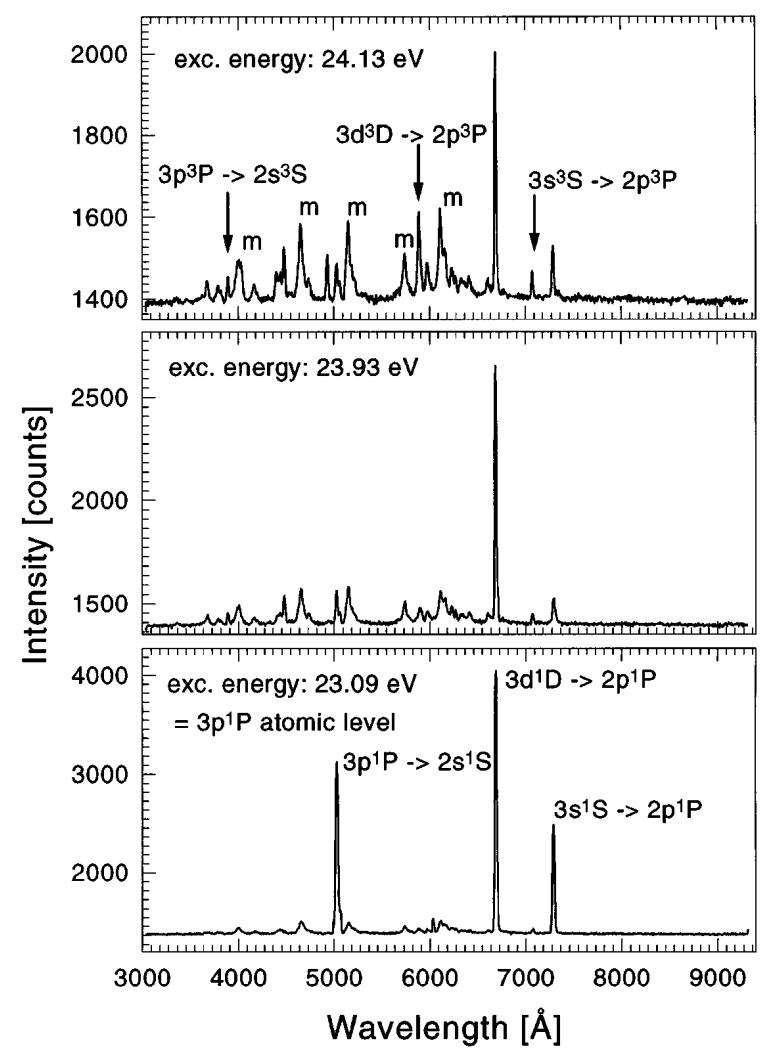

FIG. 4. Fluorescence spectra of helium clusters containing 2500 atoms at different excitation energies. Increasing excitation energy leads to luminescence from high lying levels and population of triplet states. 
In addition, no intercombination lines have been observed. Therefore, we assume that triplet states are formed during the nonradiative relaxation process. The triplet lines appear at excitation energies above $23.1 \mathrm{eV}$. At $24.55 \mathrm{eV}$ excitation energy which is very close to the vertical ionization limit [9] of large clusters the triplet lines are even stronger than the singlet lines (not shown).

The population of triplet states could result from a recombination of $\mathrm{He}_{2}^{+}$[13] either directly or dissociatively leading to the formation of $\mathrm{He}^{*}$ or $\mathrm{He}_{2}^{*}$ inside the cluster. This means first a molecular center becomes ionized, but later on the electron and the ion recombine inside the cluster. This process can only occur energetically above the ionization threshold of $\mathrm{He}_{2}$ centers in helium clusters. Below this energy the formation of atoms and molecules in triplet states should be very unlikely. The threshold of $23.1 \mathrm{eV}$ observed here is in agreement with the autoionization limit of $\mathrm{He}_{2}$ found by Hornbeck and Molnar, which is $22.4 \mathrm{eV}$ [14]. Recently, the autoionization of helium clusters has been investigated using photoexcitation. These values lie-depending on the cluster size-between 23.1 and $23.8 \mathrm{eV}$ [15].

The redistribution of energy can be studied with highly resolved luminescence spectroscopy. A rotationally resolved spectrum of the $F^{1} \Sigma_{u}^{+} \rightarrow B^{1} \Pi_{g}$ transition is displayed in Fig. 5. The line intensities can be utilized in order to derive rotational temperatures. With the well known spectroscopic constants [3] the rotational tempera-

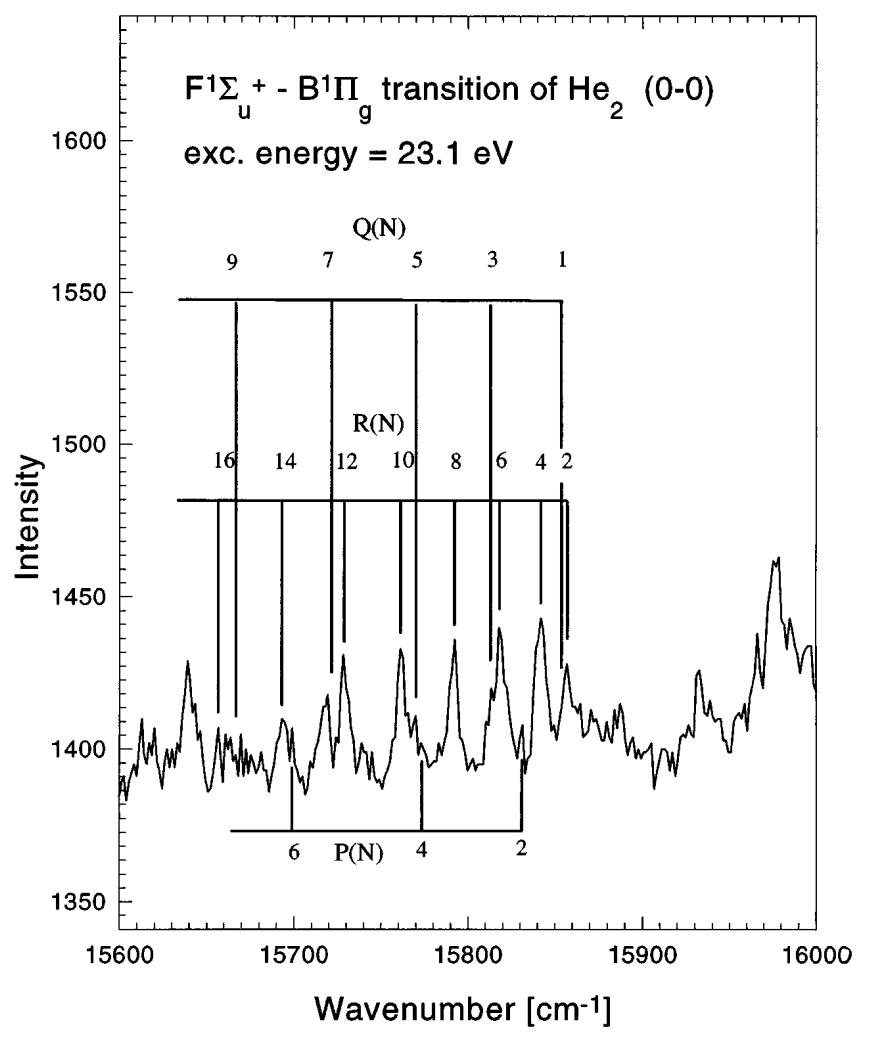

FIG. 5. Highly resolved fluorescence spectra of $\mathrm{He}_{2}$ molecules desorbing from helium clusters. Rotational energy levels of the $P, Q$, and $R$ branch are indicated. ture of molecules in the $F$-state can be estimated to $T_{\mathrm{vib}} \sim$ $450 \mathrm{~K}$ [16]. From the intensities of the hot band (1-1) the vibrational temperature is estimated to $T_{\mathrm{vib}} \sim 2500 \mathrm{~K}$. Both values are only rough estimates because a precise determination is hindered by intensity anomalies caused by perturbations of electronically excited states lying close by [3]. A comparison with the temperature of the helium clusters recently determined to $0.37 \mathrm{~K}$ [17] shows that the electronically excited molecules desorbing from the cluster are far from being thermalized. This gives strong evidence that either the coupling between the electronically excited molecule and the cluster is very weak or that the molecules leave the cluster in a very short time.

In conclusion, discrete luminescence of helium atoms and molecules desorbing from helium clusters is reported. Depending on the excitation energy, various atomic and molecular singlet and triplet states are involved in the relaxation process. With increasing cluster size relaxation processes involving molecular states become dominant. Emissions from triplet states are observed only for excitation energies above the adiabatic ionization potential of helium molecules. The vibrational and rotational temperature of helium molecules desorbing from the clusters is much higher than the temperature of the cluster itself, indicating that these molecules do not thermalize on the time scale of the radiative lifetime.

*Electronic address: haeften@desy.de

[1] W. S. Dennis, E. Durbin, W. A. Fitzsimmons, O. Heybey, and G. K. Walters, Phys. Rev. Lett. 23, 1083-1086 (1969).

[2] J. W. Keto, F. J. Scoles, M. Stockton, and W. A. Fitzsimmons, Phys. Rev. A 10, 887 (1974).

[3] M. L. Ginter, J. Mol. Spectrosc. 17, 224 (1965).

[4] K. B. Whaley, Int. Rev. Phys. Chem. 13, 41-84 (1994).

[5] R. Karnbach, M. Joppien, J. Stapelfeldt, J. Wörmer, and T. Möller, Rev. Sci. Instrum. 64, 2838-2849 (1993).

[6] O. F. Hagena, Z. Phys. D 4, 291 (1987).

[7] N. Schwentner, E.E. Koch, and J. Jortner, Electronic Excitations in Condensed Rare Gases (Springer, Berlin, 1985).

[8] M. Joppien, R. Karnbach, and T. Möller, Z. Phys. D 26, 175 (1993).

[9] M. Joppien, R. Karnbach, and T. Möller, Phys. Rev. Lett. 71, 2654 (1993).

[10] W. C. Martin, J. Res. Natl. Bur. Stand. Sect. A 64, 19 (1960).

[11] T. Kloiber and G. Zimmerer, Phys. Scr. 41, 962 (1990).

[12] V. Guzielski et al., Chem. Phys. Lett. 179, 243 (1991).

[13] S. L. Gubermann, in Physics of Ion-Ion and ElectronIon Collisions, edited by F. Brouillard NATO Advanced Study Institutes, Ser. B, Vol. 83 (Plenum Press, Baddeck, Canada, 1981), pp. 167-200.

[14] J. A. Hornbeck and J.P. Molnar, Phys. Rev. 84, 621 (1951).

[15] R. Fröchtenicht et al., J. Chem. Phys. 104, 2548 (1996).

[16] G. Herzberg, Spectra of Diatomic Molecules (Van Nostrand Reinhold, Canada, 1950), p. 124.

[17] M. Hartmann et al., Phys. Rev. Lett. 75, 1566 (1995). 